BERICHT

\section{Gegen die}

\section{politische Immobi-}

lisierung durch

Techno-Mythen

\author{
Workshop Feminist Philosophy \\ of Technology
}

Julia Valeska Schröder, Goethe Universität Frankfurt (jschroder@posteo.de)

Die Stärke feministischer Technikphilosophie, alternative Szenarien der Technikentwicklung und Existenzformen denkbar und realisierbar zu machen, sowie ,Menschheit' und Technologien herrschaftskritisch umzuschreiben, könnte eine erfrischende Inspirationsquelle für TA darstellen. Hierzu lieferte der Workshop zur feministischen Philosophie der Technik, organisiert von Janina Loh und Mark Coeckelbergh an der philosophischen Fakultät der Universität Wien vom 25. bis 26. Oktober 2018, reichhaltige theoretische und praxisorientierte Grundlagen, u. a. gegen eine politische Immobilisierung von Akteuren im Bereich der Technikreflexion, für eine Korrektur von Hypes aber auch für die Möglichkeit einer emanzipatorischen Technikgestaltung.

Obwohl die Benennung, feministische Technikphilosophie wegen ihrer disziplinären Engführung nicht alle Beteiligten überzeugen konnte, war das gemeinsame Ziel der am Workshop Teilnehmenden unstrittig: das Verhältnis von Emanzipation und Technologie(n) näher zu beleuchten und mit kritisch-theoretischen Mitteln der feministischen Technikphilosophie Herrschaftsstrukturen auf konzeptioneller, materieller, intersubjektiver und gesellschaftlicher Ebene aufzudecken. Mit Nachdruck wurde dabei demonstriert, wie feministische Theorie die notwendige Problematisierung von gegenwärtigen Technikentwicklungen in Bezug auf ihre Durchdrungenheit von gewaltträchtigen Strukturen ermöglicht und neue kritische Reflexionsräume zum Mensch-Maschine-Verhältnis öffnen kann.

This is an article distributed under the terms of the Creative Commons Attribution License CCBY 4.0 (https://creativecommons.org/licenses/by/4.0/)

https://doi.org/10.14512/tatup.28.1.77

\section{Dekonstruktion des ,menschlichen Subjekts“}

Beiträge des Workshops setzten sich aus der Perspektive eines kritischen Posthumanismus mit Automatisierung, der Ideologie des Digitalismus sowie dem Streben nach human enhancement und dem trans-human auseinander, unter anderem fokussierten sie auf Anthropozentrismus, Speziesismus, Eurozentrismus und patriarchale Machtstruktur als inhärente Charakteristika des humanistischen Menschenbildes. In vielen Vorträgen schwang keine geringere Frage mit als jene, was es bedeute, Mensch zu sein - insbesondere im Kontext technowissenschaftlicher Kulturen, des digitalen Spätkapitalismus, des Klimawandels und hochmoderner Biopolitik. Diesbezüglich wurden im Workshop zwei verschiedene Strategien deutlich, um mit dem problematischen Erbe des Humanismus umzugehen: einerseits das Aufbrechen der Kategorie ,Mensch“ und des Objekt/Subjekt-Dualismus, andererseits das Festhalten an kritisch-reflektierten, ihrer Genealogie bewussten kategorialen Differenzierungen.

\section{Technologie ohne Objekte}

Eine Dekonstruktion des humanistischen ,menschlichen Subjekts ' bedeutet für Rick Dolphijn von der Universität Utrecht, sich dem Gedanken einer „technology without objects“ anzunähern. Die von ihm verfolgte Denkrichtung des ,Neuen Materialismus ' problematisiert das hegemoniale objekt-fetischisierende Denken, das besonders in den Technikwissenschaften, aber auch in geisteswissenschaftlichen Reflexionen über Technik zu finden sei. Mithilfe seiner Frage, wie Objekte zu ihrer Existenz kommen sowie eine Permanenz und soziale Wirkmacht erlangen können, adressierte Dolphijn die Gefahren der object-oriented-ontology: Diese verhindere gesellschaftliche Transformation,

\section{Feministische Theorie ermöglicht die Problematisierung von gegen- wärtigen Technikentwicklungen in Bezug auf ihre Durchdrungenheit von gewaltträchtigen Strukturen.}

indem dominante Paradigmen, z. B. des Patriarchats, der Religionen, des Kriegs und des Kapitalismus, in Objekte eingeschrieben würden und somit durch den fälschlichen Objektcharakter verfestigt würden. Im Kontrast dazu stellte Corinna Bath, Professorin für Gender, Technik und Mobilität an der Universität Braunschweig, die intersubjektive Dimension in der Objekt-Produktion und -Konstruktion heraus. Insbesondere der ingenieurwissenschaftlichen Praxis komme demnach eine besondere Verantwortung zu, um die o. g. Verfestigungen von Paradigmen in Objekten zu reflektieren und idealerweise aufzubrechen. 


\section{Welt allein aus Subjekten}

Für eine grundlegende Betonung und Erweiterung des Subjektstatus trat auch die dänische Geschlechterforscherin Nina Lykke ein. Die techno-optimistischen Diskurse des Transhumanismus würden nämlich die Entwicklung eines Humanismus im Sinne eines universal man befördern. Sie plädierte dafür, disziplinierte Körper biopolitischer Macht und globaler Nekropolitik in einem kritisch-affirmativen Modus zu begegnen, den hegemonialen Diskurs zu dekonstruieren und das humanistische Selbstverständnis ,Mensch“ aufzubrechen und zu einer Gleichberechtigung zwischen allen Existenzformen zu entwickeln.

\section{Differences that matter}

\section{Strategischer Humanismus}

Die Soziologin Lucy Suchman, Professorin für Anthropology of Science and Technology der Lancaster University, thematisierte in ihrem Vortrag eben diese Entgrenzung von Subjekt und Objekt als Vermengung von science fiction und science fact. Sie zeigte anhand von automatisierten Waffensystemen, dass Maschinen nicht in der Lage sind, menschliche Fähigkeiten wie situatives Bewusstsein und Erkenntnisvermögen zu imitieren. Durch die Vernachlässigung dieser und anderer Unterschiede zwischen Mensch und Maschine könne eine Dehumanisierung die „böse Zwillingsschwester“ des Post-Humanismus werden. Mit ihrem Anspruch, ,differences that matter“ aufzuzeigen, zielte Suchman auf eine Grenzziehung zwischen ,Subjekt' und ,Objekt", sowie zwischen ,Mensch" und ,Maschine', die im Sinne von Spivaks ,strategischen Essentialismus“ durch eine Art „strategischen Humanismus“ erreicht werden könne.

\section{Frauen und Bots als Objekte}

Auch Kathleen Richardson war überzeugt, dass diese identitären Kategorien nicht ineinander fallen dürften. Die Professorin für Ethics and Culture of Robots and AI aus Leicester kritisierte scharf die Existenz von vorgeblich sozialen , bots ' die meist auf systematische Weise feminisiert seien. Richardson argumentierte deswegen für die Abschaffung von Sexrobotern als Phänotypen des objektivierten Patriarchats. Vor allem die Zusammenhänge von Mensch-Maschine-Interaktionen und Mensch-Mensch-Interaktionen wurden hierbei hervorgehoben: Sexroboter, fast ausschließlich in Form von hypersexualisierten Frauenkörpern, würden nicht nur die Frage nach der Bedeutung von Sex und Intimität aufwerfen, sondern seien untrennbar mit dem gesellschaftlichen Problem der (kommerziellen) Sexualisierung von Frauen verbunden. Die Mechanisierung von „Frauen“ sei Teil der Objektivierung von Frauen - allerdings in einer neuen Form materialisiert und festgeschrieben.

Dass feminisierte Roboter die Robotik-Landschaft prägen, ist kaum überraschend, ist es doch einfacher, seit Jahrhunderten objektivierte Subjekte durch ein Objekt zu imitieren. Nach der Soziologin Judy Wajcman von der London School of Economics and Political Science ist es kein Zufall, dass Erwartungen an Roboter, wie Gehorsam, Unterwerfung und Dienstbarkeit, in der
Gestaltung eines weiblichen Antlitzes münden. Die Hoffnung, dass feminisierte Arbeit durch automatisierte Maschinen abgeschafft werde, sei bereits falsifiziert, denn stereotype Projektionen würden durch ,fembots “ perpetuiert.

\section{Fazit}

Weil die auf dem Workshop kritisierten Projektionen auf Technologie bis in die Wissenschaft reichen, sollten die diskutierten Problematiken auch in der Technikfolgenabschätzung Beachtung finden: Verfällt wissenschaftliche Forschung dominanten Herrschaftsformen und ideologischen, machtpolitischen, oder wirtschaftlichen Programmen, wenn, zum Beispiel, die Sprache aus den Ursprungskontexten der Technologien reproduziert wird? Was geschieht, wenn Technologien zu bloßen Objekten werden und somit unbemerkt zentrale gesellschaftspolitische Fragen verdrängen und diskriminierende Gesellschaftsstrukturen verfestigt werden? Für häufig zu wenig beachtete Anliegen, wie die Aufschlüsselung von ,soziotechnischen Zusammenhängen', eine kritische Auseinandersetzung mit der Standardisierung und Mechanisierung des Sozialen, oder Fragen nach den Qualitäten von Mensch-Mensch-Interaktionen und nach verantwortungsvollen menschlichen Gestaltungsmöglichkeiten, kann die feministische Technikphilosophie wichtige Perspektiven eröffnen.

\section{Literatur \\ Haraway, Donna (2016): Staying with the trouble. Making kin in the Chthulucene. Durham: Duke University Press.}

\section{Weitere Informationen}

Die Workshop-Beiträge werden im Band „Feminist Philosophy of Technology" der Metzler-Reihe Techno:Phil - Aktuelle Herausforderungen der Technikphilosophie durch Birgit Beck, Bruno Gransche, Jan-Hendrick Heinrichs und Janina Loh herausgegeben. 